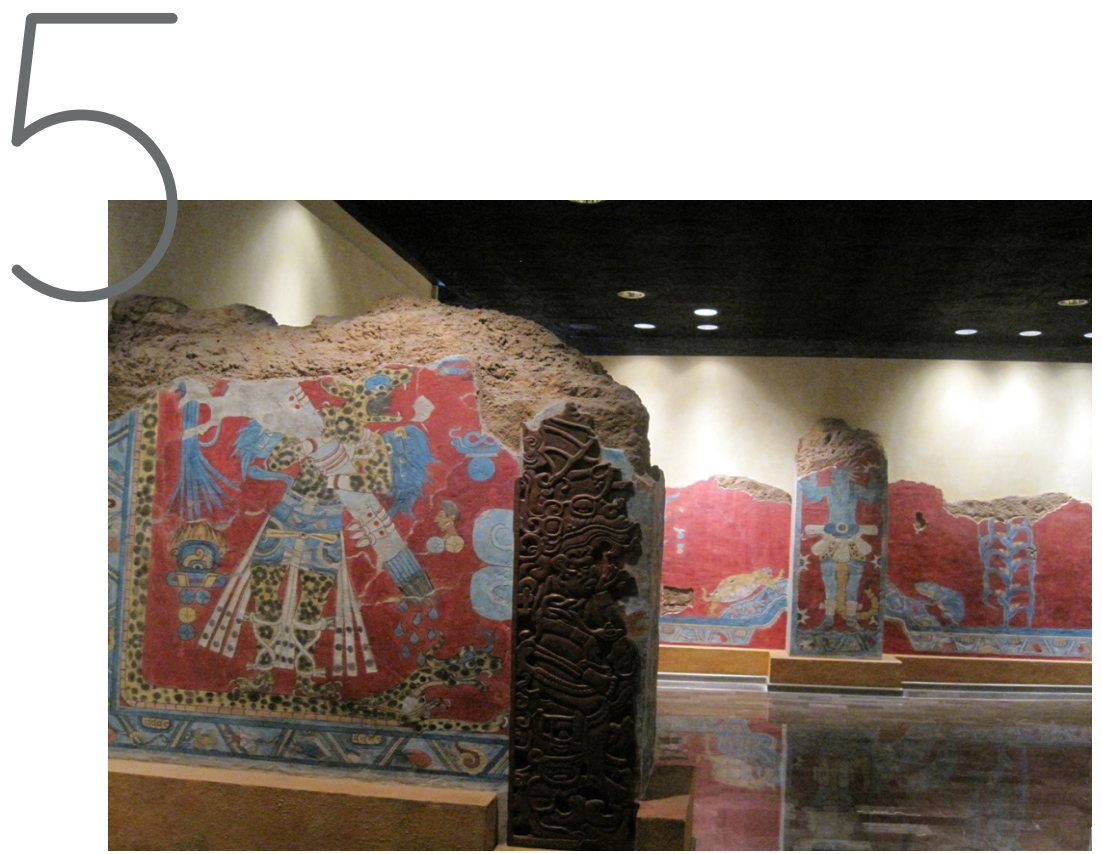

Museo de antropología de México, ciudad de México

\title{
EL MUSEO COMO PRINCIPAL ATRACTIVO DEL TURISMO CULTURAL
}

\section{Ramón Morillo, Karina1; Ullauri Donoso, Narcisa²}

1 Licenciada en Administración turística. Universidad Nacional de Loja. Magister en Planificación Turística.

2 Licenciada en Comunicación Social, Magister en Educomunicación. Máster en Estudios de la Cultura. 


\section{Resumen}

El artículo hace un análisis del museo como principal elemento dentro de la oferta del turismo cultural, porque cumple la función de revitalizador de la memoria individual y colectiva. La visión contemporánea del museo, como el espacio oportuno para el desarrollo de actividades que propicien la concientización de la cultura, además de apoyar las actividades históricas y artísticas que fomenten el crecimiento cultural de la comunidad en general, donde todas las identidades se sientan representadas. Es el lugar propicio para mostrar al turista el patrimonio tangible e intangible de la comunidad receptora.

Palabras claves: Turismo cultural, patrimonio, memoria, espacios públicos, patrimonio tangible, patrimonio intangible.

\section{THE MUSEUM AS MAIN ATTRACTION OF THE CULTURAL TOURISM}

\section{Abstract}

The article makes an analysis of the museum as the main element in the cultural tourism offer, fulfilling this function revitalization of individual and collective memory. The contemporary view of the museum, as appropriate for the development of activities that promote awareness of culture, in addition to supporting the historical and artistic activities that encourage cultural growth of the community, where all identities represented sitting space. Being the right place to show tourists the tangible and intangible heritage of the host community.

Keywords: Cultural tourism, heritage, memory, public spaces, tangible assets, intangible assets. 


\section{Turismo cultural}

Desde un punto de vista conceptual, el turismo cultural tiene su origen en la Cultura cuyo consumo vertebra, de hecho, todas las parcelas de nuestra vida cotidiana bajo múltiples formas (patrimonio, lengua, hábitos, creaciones artísticas, tradiciones, gastronomía). Pero a ello hay que añadir el viaje que, en sí mismo, adquiere como nunca una dimensión cultural a través de las distintas experiencias que los viajeros viven a lo largo de la estancia. Esta visión amplia del turismo cultural incorpora una dimensión de mercado y consumo, a la vez que destaca el valor del turismo cultural y de su oferta como herramienta de conservación del patrimonio. Además, desde esta perspectiva, se da protagonismo al visitante en la creación de modelos de visita, experienciales y creativas. (Molinero, 2013).

El turismo cultural actual nace unido al patrimonio. Fue en los años 60 del siglo XX, cuando en Europa, especialmente en Italia, se empezó a crear un marco teórico enfocado al patrimonio y sobre el significado de los "bienes culturales", y dentro de sus significados, se les asignó un objetivo último que era el de "ser disfrutados por parte del público", derecho que abriría el patrimonio a todos y sería el primer paso para la realización de una política hacia el turismo cultural (Salgado, 1999). La materialización de este sentir vendría poco después con la "Carta de Venecia" de 1965 a favor del patrimonio y con la Convención de 1972 de la UNESCO, creando el patrimonio mundial, lo que favorecería su protección pero incorporando la función de generación de conocimiento (París, 23 de noviembre de 1972). 
Yserá la UNESCO la que redactaría la primera definición de turismo cultural en 1976: "El Turismo Cultural es aquella forma de Turismo que tiene por objeto, entre otros fines, el conocimiento de monumentos y sitios histórico-artísticos. Ejerce un efecto realmente positivo sobre éstos en tanto en cuanto contribuye -para satisfacer sus propios fines- a su mantenimiento y protección. Esta forma de Turismo justifica, de hecho, los esfuerzos que tal mantenimiento y protección exigen de la comunidad humana, debido a los beneficios socio-culturales y económicos que comporta para toda la población implicada" (Carta del ICOMOS adoptada en Bruselas en 1976). (ICOMOS, 1976).

El turismo cultural se ha desarrollado bajo múltiples formas, una de ellas es el conocimiento de la cultura a través de la visita a los museos, como lo define el ICOM: "EI museo es una institución permanente, sin fines de lucro, al servicio de la sociedad y de su desarrollo, abierta al público que adquiere, conserva, estudia, expone y transmite el patrimonio material e inmaterial de la humanidad y de su medio ambiente con fines de educación y deleite" ((ICOM), 2007).

Las visitas culturales han sido una de las motivaciones para muchos viajeros y esta conexión se ha ido haciendo más nítida a partir del Renacimiento y, sobre todo, con los denominados viajes del grand tour. Estos últimos viajes son citados como el antecedente del turismo cultural por la OMT-UNESCO (OMT-UNESCO, 1993), si bien el acercamiento, al menos simbólico, entre la cultura y el turismo se produce realmente por ejemplo en España a nivel institucional, cuando en 1900 el conde de Romanones unifica, en el Ministerio de Fomento, las competencias de patrimonio y de turismo, iniciando la catalogación de 
las riquezas históricas y creando en 1905 la "Comisión Nacional de Turismo", origen de la administración turística española.

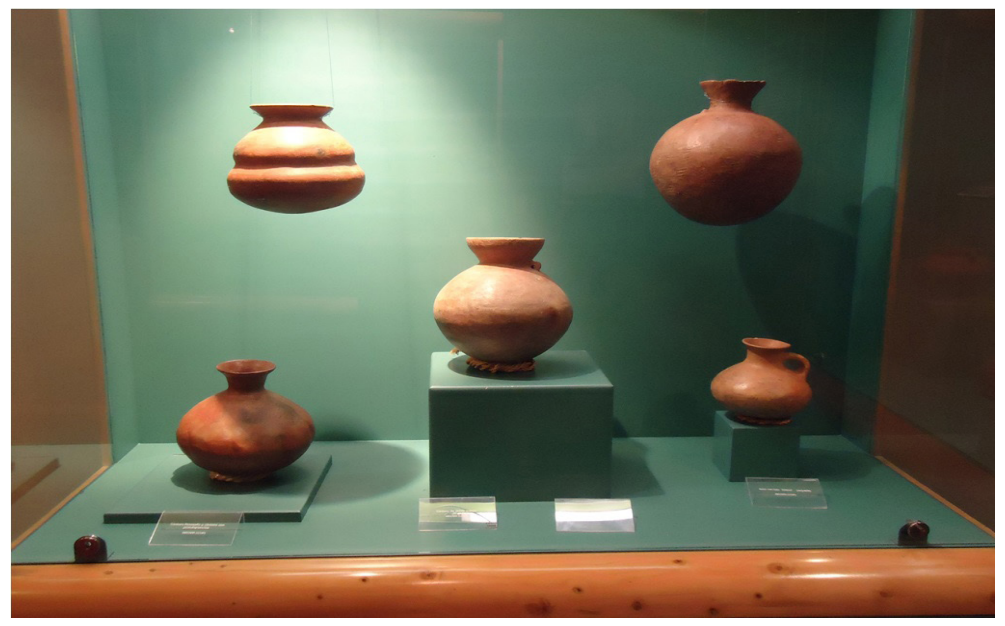

Museo de la cultura lojana

\section{El patrimonio cultural y la revitalización de la memoria}

La Carta Magna del año 2008, en el marco de la recuperación de la soberanía y la democratización, abandona el paradigma del patrimonio como un tesoro rescatado del pasado remoto, que ha devenido en mercancía, y también la noción de cultura como un signo de distinción artística de las vanguardias elitistas intelectuales de la partidocracia. En su lugar, concibe al patrimonio como una memoria activada en las relaciones sociales contemporáneas y reconoce a la creación como uno de los derechos culturales de todas las colectividades sociales y los ciudadanos ecuatorianos (art. 377). 
En este marco, la urgencia de rescatar el patrimonio cultural dio lugar al Decreto de Emergencia Patrimonial, a partir del cual se realizó un levantamiento inicial de bienes muebles e inmuebles patrimoniales y se estableció el Sistema de Información para la Gestión del Patrimonio Cultural, denominado $A B A C O$, el cual ha permitido el registro continuo del patrimonio. Actualmente existen aproximadamente 142212 inventarios y registros en el sistema (INPC, 2012: 37). También se lanzó en el año 2013 el Atlas de Infraestructura y Patrimonio Cultural del Ecuador, a través del cual la ciudadanía puede realizar consultas interactivas. Para asegurar una gestión responsable y participativa del patrimonio es necesario fortalecer la institucionalidad territorial y racionalizar la estructura de responsabilidades institucionales; de esa manera se evita la desarticulación de la política del sector. La gestión del patrimonio nunca ha estado a cargo del Ministerio de Cultura, el Decreto de Emergencia Patrimonial de 2008 fue ejecutado en su totalidad por el Ministerio Coordinador de Patrimonio.

En este contexto, la responsabilidad del Estado de garantizar el acceso al patrimonio debe estar orientada hacia el fortalecimiento de redes de investigación, bibliotecas, archivos, museos y sitios patrimoniales a nivel nacional. Estos deben operar a través de un subsistema de memoria y patrimonio por medio de instituciones nacionales que garanticen su acceso y circulación. El fin es garantizar la protección, conservación, salvaguarda y difusión de los patrimonios como un bien común. La identificación de los bienes de especial relevancia patrimonial, el cumplimiento de la agenda de emergencia patrimonial y la gestión del patrimonio contemporáneo se deben realizar con la institucionalidad y los recursos necesarios para su responsable ejecución. 


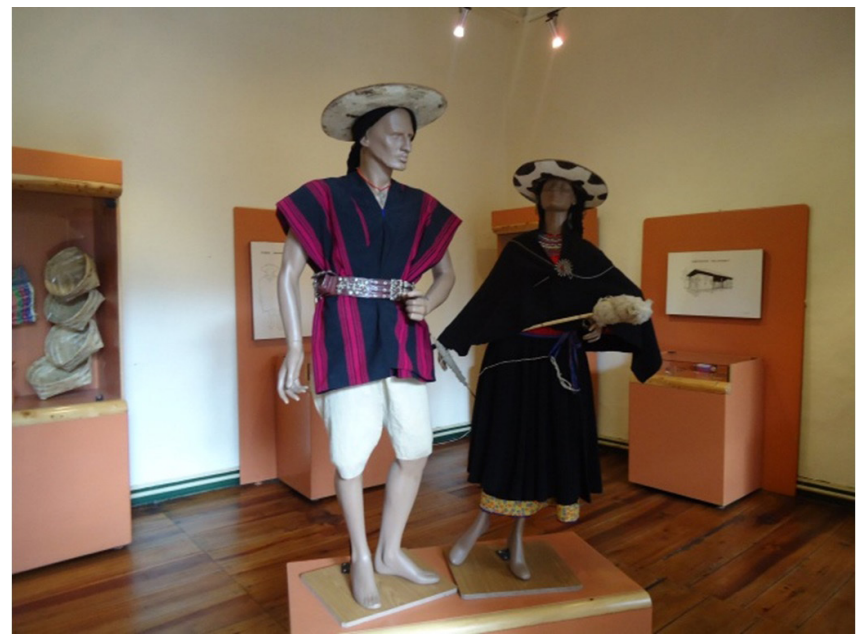

\section{El museo como parte de la estructura de atractivos en los destinos de turismo urbano}

Los museos se han creado con la finalidad de incentivar la concientización de la cultura además de apoyar las actividades históricas y artísticas que fomenten el crecimiento cultural de la comunidad en general.

Los grandes museos han sido siempre destino del turismo cultural, igual que lo han sido los monumentos, los edificios históricos o las ciudades históricas. De hecho, el turismo de las ciudades, aunque también puede ser comercial o gastronómico, muy a menudo es un turismo cultural.

Los museos como parte de los equipamientos culturales en un destino pueden capitalizar oportunidades para atraer más turistas (Borg, 2002), incluyendo a aquellos 
que no son significativamente motivados por la cultura. Aún más, los museos pueden también agregar valor a la experiencia turística, especialmente cuando los visitantes perciben en ellos la idea de localidad o autenticidad del destino. En este sentido, las directivas del museo deben reconocer que sus organizaciones son parte del mercado turístico y esto implica que sean diferenciados en términos de mercadotecnia (Rentschler, 2002). Todo parece indicar que los museos han adoptado las recomendaciones propuestas en las diferentes reuniones del ICOM, donde se ha reconocido que los museos complementan la oferta del producto cultural en un destino. Sin embargo, para considerar un museo como atractivo turístico, éste debe tener el carácter de un producto viable para el consumo turístico.

Los museos se han convertido en la atracción principal para los turistas en muchas ciudades. Es así como la cultura se vuelve un componente importante de la ciudad, y el museo, convertido en un hito de referencia, pasa a ser un elemento que puede ayudar a definir el producto turístico general del destino (Milne, 1999).

Los museos tienen que involucrarse más en los temas relacionados con el turismo, no sólo para estar en condiciones de ejercer una influencia en los encargados de la adopción de decisiones en el plano económico y gubernamental a la hora de planear políticas, sino también para llegar a los turistas de forma más directa (UNESCO, 2007).

En función del desarrollo de la actividad turística cultural local y dado que los museos tienen que competir ampliamente con otras actividades de ocio, es importante 
que estos cuenten con herramientas innovadoras para la gestión del producto turístico cultural que ofertan, que les permitan, entre otros aspectos, obtener una ventaja diferencial sostenible frente a otras alternativas turísticas y conocer las motivaciones y los patrones de comportamiento de los visitantes de los museos. Resultados éstos que pueden ofrecer claves interesantes al momento de desarrollar un producto turístico urbano atractivo (Aguirre, 2012)

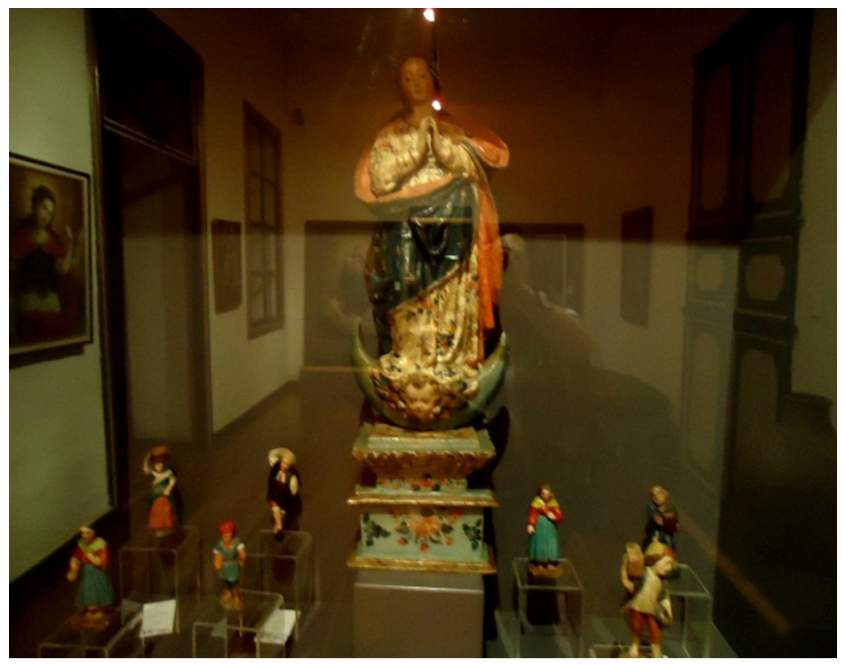

Museo de la sociedad actual

\section{El museo en la sociedad actual}

Desde hace ya un par de décadas que en los países desarrollados los centros culturales - museos constituyen las obras arquitectónicas más significativas. Contenido 
y continente son objeto de los mayores esfuerzos de imaginación, desde las más variadas perspectivas tecnológicas y disciplinarias. Progresivamente esta tendencia se ha ido también generalizando en nuestros países. $Y$ todo parece señalar que, aquí y allá, esta propensión sólo se encuentra en sus inicios. Por tamaño, ingenio, esfuerzo presupuestario y apuestas a la innovación tecnológica, día a día cada nuevo emprendimiento compite con el anterior y los "viejos" luchan por no quedar rezagados. Es en estos complejos multifacéticos y generosamente receptivos donde la sociedad quiere mostrar su mejor imagen al visitante, docentes y padres. El ciudadano común (y no tan común) busca descubrir atractivos y placidez. Esto ocurre cuando estamos viviendo no una crisis sino la mutación de toda una civilización, cuando el mayor de los capitales es el cognoscitivo y este es, simultáneamente, tan poderoso y tan efímero. Es en este contexto donde se inscribe la programación, realización, organización y gestión de un centro cultural -museo. Todos estos aspectos del emprendimiento son, demás está decirlo, tareas de equipos interdisciplinarios especializados y dedicados al proyecto, donde permanentemente se presentan desafíos fascinantes para la arquitectura y las otras especialidades intervinientes. Para una realidad que va creando nuevos espacios para el desarrollo de la imaginación, la previsión de situaciones y la variabilidad en la presentación de mensajes atractivos es la clave de todo centro cultural museo actualizado, no importa su tamaño, que siempre reúne una serie de exigencias mínimas, museográficas, científicas, educativas. (Científicas, 2002) 


\section{Conclusión}

Los museos son bienes patrimoniales, espacios públicos, elementos simbólicos identitarios de la comunidad. Son el referente para el turista, es la cara misma de la comunidad anfitriona, que ha generado elementos de identidad y conforman una personalidad diferenciada de sus habitantes.

Los museos son espacios públicos vivos desde donde se desarrolla la cultura local, representan el modo de vida de la población dueña del entorno en el que participa la población. Estos espacios propios de las ciudades se van revitalizando con un patrimonio autentico, buscando estrategias para el desarrollo local. Tienen vida propia.

Los espacios públicos laten por su gente, educan sin palabras, trasmiten una herencia cultural. El mismo hecho de tenerlos y conservarlos es la contraprestación de la población a la cultura a la que pertenecen, por ello debemos siempre buscar mantenerlos. La actividad turística se nutre de estos espacios para desarrollarse, mientras más autentico es el espacio y la actividad que en este se desarrolla, mayor afluencia de visitantes se tendrá.

El museo debe propender a fortalecer los espacios públicos buscando desarrollar actividades culturales y artísticas donde se priorice la identidad nacional a través de las memorias colectivas e individuales y el patrimonio cultural tangible e intangible.

Este reto deberá ser abordado, igualmente, de forma intersectorial, con la participación activa de todas las instituciones que comparten la responsabilidad de llevar a cabo los objetivos, las políticas y las estrategias del campo cultural, en pos del desarrollo sostenible. 


\section{Bibliografía}

Aguirre, S. Z. (2012, junio). Retrieved from www.eumed.ne.

Antigua y Medieval: Ingapirca más deterioro en las piedras. (2007, May0 11). Retrieved Mayo 27, 2011, from Antigua y Medieval: Ingapirca más deterioro en las piedras: http://antiguaymedieval. blogspot.com/2007/05/ingapirca-ms-deterioro-en-las-piedras.html

Biografías y Vidas. (n.d.). Retrieved from http://www. biografiasyvidas.com/biografia/f/francisco_asis.htm

Borg, R. y Buenaga, I. M. (n.d.). (2002). Creha. Retrieved from http://www.artecreha.com/ Iconograf\%C3\%ADa/inmaculada-concepcion-iconografia.html

Científicas, G. Y. (2002). Perspectivas del Turismo Cultural.

Cuenca Cultural. (n.d.). Retrieved from http://cuencacultural. blogspot.com/p/arqueologia-de-cuenca-dos-nuevos.html

Ecostravel. (n.d.). Retrieved from http://www.ecostravel.com/ ecuador/ciudades-destinos/museo-musica-loja.php

EWTN. (n.d.). Retrieved from http://www.ewtn.com/spanish/saints/auxiliadora_5_24.htm

ICOM. (1989, agosto 31). www.icom.museum.

ICOM, C. (1994). www.icom.com.

ICOMOS. (1976). CARTA DEL ICOMOS. Bruselas.

Ingapirca. (n.d.). Retrieved from http://ingapirca.free.fr/materiales.htm 
Lenguajes Artísticos. (n.d.). Retrieved from http://lartisticosi. blogspot.com/2011/07/escultura-colonial-en-el-ecuador.html

Milne, T. y Molina, M. (n.d.). (1999).

Monografías. Retrieved from http://www.monografias.com/ trabajos72/incas-ecuador/incas-ecuador2.shtml

Molinero, N. M. (2013). Turismo Cultural. Patrimonio, museos y empleabilidad. Madrid.

Montaño, M. (2003, Junio). Terra incognita. Retrieved from http:// www.terraecuador.net/revista_23/23_spondylus.htm

Moreno, G. y. (2009). OMT-UNESCO. (1993). org, C. (n.d.). Catholic.net. Retrieved from http://es.catholic.net/ mariologiatodoacercademaria/570/1421/articulo. php?id=4481

Ortiz, M. A. (1982). Diagnóstico de los Museos del Ecuador. Quito.

Red de Turismo Comunitario Saraguro Rikuy. (2007). Red de Turismo Comunitario Saraguro Rikuy. Retrieved Mayo 27, 2011, from Red de Turismo Comunitario Saraguro Rikuy: http://www. turismosaraguro.com/

Tarallo, M. (2012, Octubre). Retrieved Noviembre 27, 2013, from http://www.ecuadoracolores.com/ed2012_oct/pages/int02.html

UNESCO. (2007). World Heritage 
